Z Gerontol Geriat 2017 · 50:87

DOI 10.1007/s00391-016-1165-3

Online publiziert: 13. Dezember 2016

๑) Springer-Verlag Berlin Heidelberg 2016

CrossMark

\title{
R. Püllen
}

Agaplesion Frankfurter Diakonie-Kliniken, Frankfurt/Main, Deutschland

\section{Computerspiele zur Delirprävention?}

\section{Originalpublikation}

Tow A et al (2016) Cognitive reserve and postoperative delirium in older adults. J Am Geriatr Soc 64:1341-1346

Korrelieren kognitive Reserven im höheren Alter mit der Gefahr eines postoperativen Delirs? Dieser Frage ging eine prospektive Kohortenstudie nach, in die 142 kognitiv intakte, ältere $\mathrm{Pa}$ tienten (Durchschnittsalter 72 Jahre) eingeschlossen wurden. Die Patienten kamen wegen eines elektiven orthopädischen Eingriffs in die Klinik; $32 \%$ von ihnen entwickelten ein Delir. Dabei fand sich kein Zusammenhang zwischen dem Bildungsniveau der Patienten und der Delirgefahr. Dagegen waren sowohl Häufigkeit als auch Schwere eines postoperativen Delirs signifikant niedriger bei denjenigen Personen, die in den Wochen vor dem Eingriff kognitiven Aktivitäten nachgegangen waren. Von den 12 untersuchten kognitiven Aktivitäten waren dies Bücherlesen, E-MailSchreiben und Computerspielen. Bevor allerdings jetzt allgemein Computerspiele zur Delirprävention empfohlen werden, müssen diese Ergebnisse in einer randomisierten Interventionsstudie überprüft werden.

\section{Korrespondenzadresse}

PD Dr. R. Püllen

Agaplesion Frankfurter Diakonie-Kliniken Holzhausenstr. 72-92, 60322 Frankfurt/Main, Deutschland

rupert.puellen@fdk.info

Interessenkonflikt. R. Püllen gibt an, dass kein Interessenkonflikt besteht. 